\title{
Nonminimal scalar-tensor theories
}

\author{
Ismael Ayuso $\circledast^{1,2, *}$ and Jose A. R. Cembranos $\circledast^{1,3,4, \dagger}$ \\ ${ }^{1}$ Departamento de Física Teórica and IPARCOS, Universidad Complutense de Madrid, \\ E-28040 Madrid, Spain \\ ${ }^{2}$ Departamento de Física and Instituto de Astrofísica e Ciências do Espaço, Faculdade de Ciências, \\ Universidade de Lisboa, Edifício C8, Campo Grande, 1749-016 Lisbon, Portugal \\ ${ }^{3}$ Facultad de Ciencias, CUICBAS, Universidad de Colima, 28040 Colima, Mexico; \\ ${ }^{4}$ Dual C-P Institute of High Energy Physics, 28040 Colima, Mexico
}

(Received 23 September 2019; accepted 31 December 2019; published 5 February 2020)

\begin{abstract}
We compute the spectrum, mixing and interaction structure of scalar models with a general coupling to the scalar curvature. As it is well known the perturbative states of these theories are given by two massive spin-0 modes in addition to one massless spin- 2 state. This latter mode can be identified with the standard graviton field. Indeed, it is possible to define an Einstein frame, where the dynamics of the massless spin-2 graviton is the one associated with the Einstein-Hilbert action. We explore systematically the interactions of all these degrees of freedom in the mentioned frame, since part of the coupling structure can be anticipated by geometrical arguments.
\end{abstract}

DOI: 10.1103/PhysRevD.101.044007

\section{INTRODUCTION}

There are different theoretical and experimental reasons to consider gravitational theories beyond general relativity (GR) [1]. Among them, we can mention Lovelock theories [2], Gauss-Bonnet models [3], extra dimensional geometrical extensions [4], supergravity [5], nonlocal gravitational modifications [6], Lorentz violating and $C P T$ breaking models [7], vector (or higher spin)-tensor models [8], disformal gravity [9] and scalar-tensor theories (STTs) $[10,11]$. This latter case will be the particular subject of our work; i.e., we will study particular extensions of the gravitational interaction, that are defined by the addition of scalar degrees of freedom (d.o.f.). These new spin-0 states act as mediators of part of the total gravitational force.

Currently, there are only one candidate for a fundamental scalar particle. Its discovery was announced in 2012 by both ATLAS [12] and CMS [13] collaborations. It has associated a mass around $125 \mathrm{GeV}$ and it is consistent with the predictions for the so-called Higgs boson of the Standard Model (SM) of particles and interactions. However, many other scalar fields are motivated by different theories, such as the Jordan-Fierz-Brans-Dicke (JFBD) model [14] or different low energy approaches to string theory [15], where the new scalar states are typically coupled to the matter sector through the trace of the energy-momentum tensor. In such a case, thanks to a series of field redefinitions, it is possible to reexpress the

\footnotetext{
iayuso@fc.ul.pt

†cembra@fis.ucm.es
}

extended gravitational theory in terms of the Einstein gravity associated with general relativity (GR) with new couplings to the matter sector. A similar phenomenology is associated with the so-called $f(R)$ theories [16-18], whose gravitational action is defined in terms of a given function $f$, of the scalar curvature $R$. In addition, extended models with scalar fields nonminimally coupled to gravity have been proposed in the present literature. In particular, the possibility of supporting the early inflation with scalar models, which exhibits nonzero vacuum expectation values (VEVs) at low energies, has inspired the generalized Higgs inflation models (GHIMs) [19].

As we have commented, the new interactions mediated by these scalar d.o.f. can be interpreted as part of the gravitational force. In addition, the phenomenology associated with these new d.o.f. themselves can be very rich and provide viable solutions to open problems in cosmology such as the mentioned inflation, dark energy or dark matter [18]. However, these new models suffer important constraints. For instance, this new field can lead to effective variations of fundamental constants, such as gauge and Yukawa couplings or masses. In particular, precision tests of gravity, big bang nucleosynthesis (BBN) [20-25], cosmic microwave background anisotropies [26] and weak-lensing [27] constrain the phenomenology of these scalar fields. In any case, different scalar models are not very sensitive to these restrictions [17,28-31]. In this work, we analyze the biscalar content of a general nonminimal scalar tensor theory (NMSTT). We provide explicit expressions for their masses, their mixing and their coupling between them and with the Standard Model (SM) of particles. Finally we 
discuss part of the phenomenological consequences of these features, as the instabilities of GHIMs.

The paper will be organized as follows. In the next section, we will define the NMSTT which will be studied. We will provide the general set of equations that defines the Einstein frame (EF) through a conformal transformation. In Sec. III, we will report the general couplings, which are enforced by such a transformation. They will be particularized for the SM content. We will then set up our formalism for treating different gravitational theories in Sec. IV: JFBD model [14], $f(R)$ theories [16-18] and GHIMs [19]. In Sec. V, we will resume our main conclusions.

\section{NONMINIMAL SCALAR-TENSOR THEORIES}

The most general action for a scalar field nonminimally coupled to the scalar curvature $R$, associated with the space-time metric $g_{\mu \nu}$, can be written in the following form:

$$
S_{\mathrm{NMSTT}}=\frac{1}{2} \int d^{4} x \sqrt{-g}\left[-g^{\mu \nu} \nabla_{\mu} \varphi \nabla_{\nu} \varphi+J(\varphi, R)\right],
$$

if we restrict the scalar field derivatives to the standard kinetic contribution (the first term). The function $J(\varphi, R)$ takes into account the mentioned coupling, that in general, it is nonseparable. Note that this term may include a potential (mass or self interaction) for the scalar field, or a pure gravitational contribution without explicit dependence on $\varphi$. Unless otherwise specified, we will use reduced Planck units throughout this work $(\kappa \equiv \sqrt{8 \pi G}=c=\hbar=1)$, Greek indices run from 0 to 3 , and the symbol $\nabla_{\mu}$ denotes the standard covariant derivative defined with respect to the metric $g_{\mu \nu}$.

In addition, we will assume a matter content, whose fields will be represented by the letter $\mu_{i}$, minimally coupled to the space-time metric:

$$
S_{\mu_{i}}=S_{i}\left(g_{\mu \nu}, \mu_{i}\right)
$$

where by matter, we denote any field in the theory in addition to $\varphi$ and $g_{\mu \nu}$. In such a case, we can claim that we have defined the model in the Jordan frame (JF), in which the metric $g_{\mu \nu}$ couples in this standard way to the matter content. Although the action (1) did not support explicitly a kinetic term for the gravitational interaction, this could be implicit due to the presence of the Ricci scalar in the coupling $J(\varphi, R)$. In any case, the gravitational interaction between matter fields can suffer important modifications. Within the JF, this effect seems natural to be interpreted as a modification of the Newton constant by the presence of the scalar field $\varphi$, but it means that the scalar mode is mediating part of the gravitational interaction. Finally, we will show that the model given by Eq. (1) supports another scalar perturbative d.o.f., that is also coupled to the matter content and completes the gravitational force, as it was first noted in [32].

In order to clarify the spectrum of the model, it is convenient to work in the EF, that is defined by a conformal transformation, which rewrites the action for the metric in the standard Einstein-Hilbert term. In fact, two metrics $\left(g_{\mu \nu}^{*}\right.$ and $g_{\mu \nu}$ ) are conformally related if there is a function $\Omega$, which verifies:

$$
g_{\mu \nu}^{*}=\Omega^{2} g_{\mu \nu} .
$$

It implies that $g^{* \mu \nu}=\Omega^{-2} g^{\mu \nu}, \sqrt{-g}=\Omega^{-n} \sqrt{-g^{*}}$ and

$$
\begin{aligned}
R^{*}= & \Omega^{-2} R-2(n-1) g_{*}^{\mu \nu} \nabla_{\mu}^{*} \nabla_{\nu}^{*} \ln \Omega \\
& +(n-2)(n-1) g_{*}^{\mu \nu} \nabla_{\mu}^{*} \ln \Omega \nabla_{\nu}^{*} \ln \Omega,
\end{aligned}
$$

where $n$ is the dimension of the manifold, $R^{*}$ is the Ricci scalar associated with the metric $g_{\mu \nu}^{*}$, and $\nabla^{*}$ is its corresponding covariant derivative. The function $\Omega$ is dubbed the conformal factor of the transformation. We will make use of the above expressions for $n=4$. In particular, for rewriting the action, it is particularly useful the following expression:

$$
\begin{aligned}
\sqrt{-g} R= & \Omega^{-2} \sqrt{-g^{*}}\left[R^{*}+6 g_{*}^{\mu \nu} \nabla_{\mu}^{*} \nabla_{\nu}^{*} \ln \Omega\right. \\
& \left.-6 g_{*}^{\mu \nu} \nabla_{\mu}^{*} \ln \Omega \nabla_{\nu}^{*} \ln \Omega\right] .
\end{aligned}
$$

In order to identify the correct conformal transformation that defines the $\mathrm{EF}$, it is convenient to work with an auxiliary scalar field $\phi$, defined by the following equation:

$$
J(\varphi, R)=J(\varphi, \phi)+J^{\prime}(\varphi, \phi)(R-\phi),
$$

where ' denotes the partial derivative of the $J$ function with respect to its second argument:

$$
J^{\prime}(\varphi, \phi)=\partial_{\phi} J(\varphi, \phi) .
$$

We assume that $J^{\prime \prime}(\varphi, \phi) \neq 0$. We will discuss separately the case $J^{\prime \prime}(\varphi, \phi)=0$ in following sections, but we can advance that, in such a case, the auxiliary field cannot be defined. By expressing $J(\varphi, R)$ in terms of $\phi$ in the action (1), we can write a Lagrangian that is linear on $R$. Indeed, the two actions are equivalent if we also impose optimization with respect to $\phi$ in order to obtain the equations of motion, whose solution implies $\phi=R$.

Now, it is evident to identify the proper conformal factor associated with the Einstein metric, i.e., the metric corresponding to the EF:

$$
\Omega^{2}=J^{\prime}(\varphi, \phi) .
$$

where we are assuming explicitly $J^{\prime}(\varphi, \phi)>0$. This sign is necessary to guarantee a positive Newton constant, i.e., an 
attractive gravitational interaction mediated by the standard spin-2 massless graviton. In the opposite case, the graviton has a ghost character since its kinetic term has the wrong sign. Finally, we can define a new scalar $\Phi$, in terms of the fields $\varphi$ and $\phi$ :

$$
\Phi=\sqrt{3 / 2} \ln J^{\prime}(\varphi, \phi),
$$

so that, except for a boundary term, we can write the total action as:

$$
\begin{aligned}
S_{\mathrm{NMSTT}}= & \frac{1}{2} \int d^{4} x \sqrt{-g^{*}}\left[R^{*}-g_{*}^{\mu \nu} \nabla_{\mu}^{*} \Phi \nabla_{\nu}^{*} \Phi\right. \\
& \left.-g_{*}^{\mu \nu} e^{-\sqrt{\frac{2}{3}} \Phi} \nabla_{\mu}^{*} \varphi \nabla_{\nu}^{*} \varphi-2 V(\varphi, \Phi)\right],
\end{aligned}
$$

where:

$$
V(\varphi, \Phi)=\frac{1}{2}\left[\phi(\varphi, \Phi) e^{-\sqrt{\frac{2}{3}} \Phi}-J(\varphi, \phi(\varphi, \Phi)) e^{-2 \sqrt{\frac{2}{3}} \Phi}\right] .
$$

is the potential associated with the self-interaction of the scalar modes $\varphi$ and $\Phi$, and the interaction between them. One needs to use Eq. (9) in order to write $\phi$ in terms of $\varphi$ and $\Phi$. It is interesting to remark that the third term in Eq. (10) does not only account for the standard kinetic term for $\varphi$, but also for a derivative interaction with $\Phi$. This follows, for example, by expanding the exponential factor around $\Phi=0$, i.e., by assuming a small deviation from the two frames, a small difference between $g_{\mu \nu}$ and $g_{\mu \nu}^{*}$. On the other hand, the first term in (10) corresponds to the standard Einstein-Hilbert action and the second one is associated with a pure kinetic term for $\Phi$. Of course, the kinetic terms include the interaction of both scalar fields with the geometry through the metric tensor $g_{\mu \nu}^{*}$.

\section{INTERACTION WITH THE MATTER CONTENT: THE STANDARD MODEL}

It is interesting to analyze in more detail the interactions associated with the different modes contained in the spectrum of the theory. Indeed, the coupling of the scalar field $\varphi$ is open and not restricted by the geometrical structure of the model. In particular, its coupling with the SM can be assumed to be absent. The opposite situation corresponds to the other scalar field $\Phi$ and the metric tensor. As we have discussed, the matter content is explicitly coupled to $\Phi$ when the action is expressed in terms of the Einstein metric:

$$
S_{\mu_{i}}=S_{i}\left(e^{-\sqrt{\frac{2}{3}} \Phi} g_{\mu \nu}^{*}, \mu_{i}\right) .
$$

For example, we can follow [18] in order to detail the coupling of this scalar mode. This computation can be done directly with the help of the original action (JF), but it is more transparent and easier in the EF. We can study the couplings at the linear order by expanding perturbatively the Jordan metric over the Minkowski background [18]:

$$
g_{\mu \nu}=\eta_{\mu \nu}+\frac{1}{2} h_{\mu \nu}^{*}-\sqrt{\frac{2}{3}} \Phi \eta_{\mu \nu},
$$

where $h_{\mu \nu}^{*}$ takes into account the standard two d.o.f. associated with the spin-2 (traceless and divergencefree) graviton. For simplification, we assume a common Minkowski background for the Jordan and Einstein geometries. In other words, we expand around $\Phi=0$. In such a case, for computing the linear order analysis, we do not need to specify the frame for quantities such as the energymomentum tensor.

By taking variations with respect to the metric in the matter action, it is evident that the spin-2 d.o.f. will have associated the standard interaction with the corresponding energy-momentum tensor. On the other hand, the coupling of the spin-0 mode at the linear level will be given by the trace of the same energy-momentum tensor:

$$
\mathcal{L}_{\Phi-T_{\mu \nu}}=\frac{1}{\sqrt{6}} \Phi T_{\mu}^{\mu}
$$

It means that $\Phi$ interacts with massive SM fields at tree level. In particular, the three body couplings are given by:

$$
\begin{aligned}
& \mathcal{L}_{\Phi-\mathrm{SM}}^{\text {tree-level }}=\frac{1}{\sqrt{6}} \Phi\left[2 m_{h}^{2} h^{2}-\nabla_{\mu}^{*} h \nabla_{*}^{\mu} h\right. \\
& \left.+\sum_{\psi} m_{\psi} \bar{\psi} \psi-2 m_{W}^{2} W_{\mu}^{+} W^{-\mu}-m_{Z}^{2} Z_{\mu} Z^{\mu}\right],
\end{aligned}
$$

with the Higgs boson (h), (Dirac) fermions $(\psi)$, and electroweak gauge bosons ( $W^{\mu}$ and $Z^{\mu}$ ), respectively. In addition, this scalar field interacts with photons and gluons by radiative corrections induced at one loop by charged gauge bosons and fermions (i.e., due to the conformal anomaly [18]):

$\mathcal{L}_{\Phi-\mathrm{SM}}^{\text {one-loop }}=\frac{1}{\sqrt{6}} \Phi\left[\frac{\alpha_{\mathrm{EM}} c_{\mathrm{EM}}}{8 \pi} F_{\mu \nu} F^{\mu \nu}+\frac{\alpha_{\mathrm{S}} c_{\mathrm{G}}}{8 \pi} G_{\mu \nu}^{a} G_{a}^{\mu \nu}\right]$,

where $F_{\mu \nu}$ is the gauge invariant electromagnetic field strength tensor, $G_{\mu \nu}^{a}$ represents the gluon field strength tensor, $\alpha_{\mathrm{EM}}$ is the fine-structure constant, and $\alpha_{s}$ is the strong coupling constant. The particular value of the couplings $c_{\mathrm{EM}}$ and $c_{\mathrm{G}}$, depends on the energy and the complete set of particles charged with respect to these gauge interactions.

\section{SCALAR SPECTRUM}

Another general property given by the geometrical structure of the model is that the kinetic term for $\varphi$ has the same coupling with $\Phi$ as the matter fields. Indeed, $\Phi$ can be understood as a dilaton, that parametrizes the 
conformal factor and couples to the trace of the energymomentum tensor. However, there is a general mixing of the scalar sector of the theory trough the mass matrix that is defined by the potential function $V(\varphi, \Phi)$. If we assume that this potential reaches a minimum of value $V_{0}$ at $\left(\varphi_{0}, \Phi_{0}\right)$ (i.e., $\left.V\left(\varphi_{0}, \Phi_{0}\right)=V_{0}\right)$, the squared-mass matrix (SMM) is given by:

$M_{\varphi \Phi}^{2}=\left.\left(\begin{array}{cc}e^{\sqrt{\frac{2}{3}} \Phi_{0}} \partial_{\varphi \varphi}^{2} V(\varphi, \Phi) & e^{\sqrt{\frac{1}{6}} \Phi_{0}} \partial_{\varphi \Phi}^{2} V(\varphi, \Phi) \\ e^{\sqrt{\frac{1}{6}} \Phi_{0}} \partial_{\Phi \varphi}^{2} V(\varphi, \Phi) & \partial_{\Phi \Phi}^{2} V(\varphi, \Phi)\end{array}\right)\right|_{\left(\varphi_{0}, \Phi_{0}\right)}$.

Therefore, the mass eigenstates cannot be generally identified either with $\varphi$ or with $\Phi$, but with a linear mixing of both. This fact is not in contradiction with the dilaton nature of the couplings associated with $\Phi$. The question is that the SMM breaks, in general, scale invariance explicitly.

In any case, a non trivial mixing and even the presence of two scalar d.o.f. is not completely general. There are particular forms or values of the function $J(\varphi, \phi)$ that are interesting to analyze separately as we do in the following paragraphs.

\section{A. Linear couplings}

In the particular case of a linear coupling of the field $\varphi$ with the Ricci scalar, the action (1) has associated a truncated scalar spectrum. Indeed, in such a case, we can write $J(\varphi, R)$ in terms of two functions of $\varphi$ : the one that parametrizes its nonminimal interaction $H(\varphi)$, and the one that defines its potential $U(\varphi)$ :

$$
J(\varphi, R)=H(\varphi) R-2 U(\varphi) .
$$

In this case, it is not necessary to introduce any additional scalar field, since it is possible to define a conformal transformation to the EF, through a conformal factor that depends only on $\varphi$, namely:

$$
\Omega^{2}=H(\varphi) .
$$

Following for example [11], if we redefine the scalar field in the following way:

$$
\frac{1}{2 H(\varphi)}=\frac{1}{2}\left(\frac{d \varphi_{*}}{d \varphi}\right)^{2}-\frac{3}{4}\left(\frac{d \ln H(\varphi)}{d \varphi}\right)^{2},
$$

we can write the action in the EF with a standard kinetic term for $\varphi_{*}$ :

$S_{\mathrm{NMSTT}}=\int d^{4} x \sqrt{-g^{*}}\left[\frac{1}{2} R^{*}-\frac{1}{2} g_{*}^{\mu \nu} \nabla_{\mu}^{*} \varphi_{*} \nabla_{\nu}^{*} \varphi_{*}-V\left(\varphi_{*}\right)\right]$, where the potential for $\varphi_{*}$ takes into account the conformal factor and the field redefinition:

$$
V\left(\varphi_{*}\right)=\frac{U\left(\varphi\left(\varphi_{*}\right)\right)}{H^{2}\left(\varphi\left(\varphi_{*}\right)\right)} .
$$

By assuming a minimum for this potential at $\varphi_{*}^{0}$, the corresponding squared-mass of the scalar mode will be given by:

$$
m_{\varphi_{*}}^{2}=\left.\partial_{\varphi_{*} \varphi_{*}}^{2} V\left(\varphi_{*}\right)\right|_{\varphi_{*}^{0}} .
$$

This simple example illustrates the complexity in the identification of the scalar states. In this case, it is the field $\varphi$ (or $\varphi_{*}$ ), the one that has associated the dilaton couplings with the matter content, since the conformal transformation is parametrized by $\varphi$ (or $\varphi_{*}$ ):

$$
S_{\mu_{i}}=S_{i}\left(\frac{g_{\mu \nu}^{*}}{H\left(\varphi\left(\varphi_{*}\right)\right)}, \mu_{i}\right) .
$$

\section{B. $f(R)$ theories}

Other simple examples of models described by Eq. (1) are the so-called $f(R)$ theories. In this case, the $J(\varphi, R)$ can be written as the sum of two functions, one depending on $R$ and another one depending on $\varphi$ :

$$
J(\varphi, R)=f(R)-2 U(\varphi) .
$$

The first one gives the name to these models and the second one constitutes a standard potential for the scalar field $\varphi$. In this case, $\varphi$ is minimally coupled to gravity, and it can be interpreted as part of the matter content. However, the nonlinear dependence on $R$ introduces an additional scalar d.o.f. Here, we can just particularize the equations derived in the previous section for the general case. In fact, the new scalar field, when properly normalized, is defined by

$$
\Phi=\sqrt{3 / 2} \ln f^{\prime}(\phi),
$$

where the auxiliary field $\phi$, verifies $\phi=R$ by taking into account the equations of motion. Here, ' means the derivative with respect to the unique argument $\phi$. The corresponding conformal factor is

$$
\Omega^{2}=f^{\prime}(\phi) .
$$

Note that the total potential for the scalar sector cannot be written in general as the sum of two individual potentials associated with each one of the fields:

$$
\begin{aligned}
V(\varphi, \Phi)= & \frac{1}{2}\left[\phi(\Phi) e^{-\sqrt{\frac{2}{3}} \Phi}-f(\phi(\Phi)) e^{-2 \sqrt{\frac{2}{3}} \Phi}\right. \\
& \left.+2 U(\varphi) e^{-2 \sqrt{\frac{2}{3}} \Phi}\right]
\end{aligned}
$$


The reason is that the conformal factor introduces a nonderivative interaction between the two scalar modes. Therefore, even in this case, the off-diagonal entries of the SMM are not necessarily zero and the mass eigenstates cannot be identified, in general, with $\varphi$ or with $\Phi$.

\section{Generalized Higgs inflation models}

Nonminimal gravitational couplings of the SM Higgs doublet have been considered in order to build viable models of inflation in the early universe [33]. Generalizations of this idea with nonlinear couplings to the Ricci scalar have been discussed in the literature with the SM Higgs working as inflaton or with a similar scalar field [19]. As far as we know, the presence of a new scalar d.o.f. has been missed in these analyses. As we have shown, the nonlinear couplings in the Ricci scalar introduce a new d.o.f. We can determine its phenomenology by using the general equations deduced in this work. The general $J(\varphi, R)$ function that defines these models can be written as

$$
J(\varphi, R)=R+\xi \varphi^{a} R^{b}-2 U(\varphi),
$$

where the first term is associated with the initial EinsteinHilbert action for the Jordan metric; the second term is the nonlinear coupling parametrized by the strength constant $\xi$ and the exponents $a$ and $b$; and $U(\varphi)$ is the potential for the scalar field in the JF. Note that $a$ and $b$ need to be integer numbers to have an analytical interaction at $\varphi=0$ and $R=0$ respectively, but the nonminimal coupling can be also defined for any real value of both exponents. The standard potential in these models is usually assumed to be

$$
U(\varphi)=\frac{\lambda}{4}\left(\varphi^{2}-\frac{\mu^{2}}{\lambda}\right)^{2},
$$

with $\mu, \lambda>0$. It implies that $U(\varphi)$ is bounded from below, and develops a stable minimum at $\varphi_{0}=\mu / \sqrt{\lambda}$ with $U\left(\varphi_{0}\right)=0$, i.e., we have avoided the introduction of a vacuum energy. ${ }^{1}$ By taking into account our previous results, the conformal transformation to the EF is defined by

$$
\Omega^{2}=1+\xi b \varphi^{a} \phi^{b-1},
$$

with $\phi=R$ as we have commented. Therefore, provided $b \neq 1$, the coupling introduces a new scalar particle, associated with the normalized field:

$$
\Phi=\sqrt{3 / 2} \ln \left(1+\xi b \varphi^{a} \phi^{b-1}\right) .
$$

As we have discussed, this new d.o.f. is associated with the dynamics of the Jordan Ricci scalar through the relation:

\footnotetext{
${ }^{1}$ The potential develops an analogous minimum at $\varphi_{0}=-\mu / \sqrt{\lambda}$. The same discussion applies when the system chooses this other vacuum state.
}

$R=\phi=\left\{[\exp (\sqrt{2 / 3} \Phi)-1] /\left(\xi b \varphi^{a}\right)\right\}^{1 /(b-1)}$. Therefore, the total action for this type of GHIMs is written in the $\mathrm{EF}$ as:

$$
\begin{aligned}
S_{\mathrm{GHIM}}= & \frac{1}{2} \int d^{4} x \sqrt{-g^{*}}\left[R^{*}-g_{*}^{\mu \nu} \nabla_{\mu}^{*} \Phi \nabla_{\nu}^{*} \Phi\right. \\
& \left.-g_{*}^{\mu \nu} e^{-\sqrt{\frac{2}{3}} \Phi} \nabla_{\mu}^{*} \varphi \nabla_{\nu}^{*} \varphi-2 V(\varphi, \Phi)\right],
\end{aligned}
$$

where:

$V(\varphi, \Phi)=\frac{1}{2}\left[\xi \varphi^{a}(b-1)\left(\frac{e^{\sqrt{\frac{2}{3}} \Phi}-1}{\xi b \varphi^{a}}\right)^{\frac{b}{b-1}}+2 U(\varphi)\right] e^{-2 \sqrt{\frac{2}{3}} \Phi}$.

In order to simplify the discussion, we can fix the particular values: $a=b=2$, and $\xi>0$. In such a case, the total potential is bounded from below provided $U(\varphi)$ is it as well. Indeed, a minimum of the scalar sector can be found at $\left(\varphi_{0}, \Phi_{0}\right)=(\mu / \sqrt{\lambda}, 0)$ if the potential (30) is assumed. In fact, such a minimum is global since $V\left(\varphi_{0}, \Phi_{0}\right)=0$ and $V(\varphi, \Phi)$ is non-negative. In this case, the SMM around the minimum is given by:

$$
M_{\varphi \Phi}^{2}=\left(\begin{array}{cc}
2 \mu^{2} & 0 \\
0 & \frac{\lambda}{6 \xi \mu^{2}}
\end{array}\right) .
$$

Therefore, in this particular case, the mass eigenstates can be identified with $\varphi$ and $\Phi$. In other words, they do not mix. In addition, the phenomenology of $\Phi$ is decoupled since the mass scale $\mu$ is expected to be very small with respect to the Planck scale, what implies a very large mass for $\Phi$ (by assuming $\lambda, \xi \sim 1$ ).

It is important to comment that the original action has a parity symmetry associated with the sign of the scalar $\varphi$. However this is not the fundamental reason for the nonmixing of the scalar fields, since this discrete $\mathbb{Z}_{2}$ symmetry is broken by the $\operatorname{VEV}$ of $\varphi$. It means that this symmetry will not be able to protect this property, that will be potentially destroyed by radiative corrections.

Coming back to the on-shell analysis, the situation is different for $b=2$ and $a<-2$. In this case, $\Phi$ is lighter than $\varphi$ for $\mu \ll 1(\lambda, \xi \sim 1)$. Indeed, for such values of the exponents, the SMM is still diagonal, but the nonzero entries are $M_{\varphi \varphi}^{2}=2 \mu^{2}$ and $M_{\Phi \Phi}^{2}=\lambda^{a / 2} /\left(6 \xi \mu^{a}\right)$. Finally, we must mention that any other integer value of $b(b>2)$ may imply strong instabilities for the field configuration defined by $\left(\varphi_{0}, \Phi_{0}\right)=(\mu / \sqrt{\lambda}, 0)$, since the potential will develop a singularity at $\Phi=0$. Indeed, the study of the SMM deduced in this work within the EF, is the most efficient way to analyze the stability of a NMSTT (in the same way as for $f(R)$ theories, as it was originally pointed out in [17]). This procedure is equivalent to the Hessian matrix analysis of an optimization study in two variables. 


\section{DISCUSSION}

In this work, we have studied the phenomenology of NMSTTs, i.e., scalar field models defined by a general coupling of the scalar field with the Ricci scalar. These theories can be understood as generalizations of the gravitational interaction written in a particular JF. We have found explicitly the EF corresponding to such general theories by characterizing the conformal transformation that defines the relation between both frames. By following the general set of equations associated with the transformation between the two corresponding metric tensors, it is explicit that the spectrum of the theory contains not one, but two (generally massive) scalar d.o.f., in addition to a massless spin-2 state. The latter particle can be associated with the standard mediator of Einstein gravity, whereas the second scalar mode is related to the nonlinear coupling of the original (JF defined) scalar field to the Ricci scalar. Indeed, provided that the coupling is linear, we have proved that the general conformal transformation is not well defined and the spectrum of the theory is truncated by removing the second scalar d.o.f. The situation is more involved if the Jacobian associated with the field redefinition is zero for particular values of the fields. In such a case, the effective number of d.o.f. of the theory depends on the field configuration. This fact can be understood as if the spectrum of the theory maximizes the number of perturbative states, but the masses of the scalar modes depend on the values of the fields and can diverge for one of the modes. This fact removes effectively one of the scalar d.o.f. as it was discussed in $[17,18]$ for the case of $f(R)$ theories.
Once the d.o.f. were identified, we have analyzed their couplings with the matter content. In particular, we have studied the couplings with SM particles. Under general assumptions, the spin- 2 state couples as the standard GR graviton. The scalar d.o.f. associated with the conformal factor couples through the trace of the energy-momentum tensor. Indeed, it can be identified with a dilaton since it parametrizes general scale transformations. On the other hand, the coupling of the other spin- 0 particle is completely model dependent. It changes by depending on the definition of its interactions in the original action (JF), that is not fixed. It is interesting to remark that this factorization of the couplings associated with the scalar content of the theory is simple because it is discussed in terms of the interaction eigenstates. These modes are not necessarily the mass eigenstates. In general, there is a mixing between the two scalar modes that leads to the rich phenomenology associated with these NMSTTs.

\section{ACKNOWLEDGMENTS}

We are thankful to J. Beltran Jimenez and A. de la Cruz-Dombriz for very useful comments. This work has been supported by MICINN and MINECO (Spain) projects No. FIS2011-23000, No. FPA2011-27853-C0201, No. FIS2014-52837-P and No. FIS2016-78859-P (AEI/FEDER, UE), Consolider-Ingenio MULTIDARK CSD2009-00064, the Grant No. PD/BD/114435/2016 from IDPASC and FCT/MCTES through national funds (PIDDAC) by the Grant No. UID/FIS/04434/2019 and by the Project No. PTDC/FIS-OUT/29048/2017.
[1] A. Dobado and A. L. Maroto, Phys. Lett. B 316, 250 (1993); 321, 435(E) (1994); S. Nojiri and S. D. Odintsov, eConf C0602061, 06 (2006); Int. J. Geom. Methods Mod. Phys. 04, 115 (2007); Phys. Rep. 505, 59 (2011); S. Capozziello and M. Francaviglia, Gen. Relativ. Gravit. 40, 357 (2008); T. P. Sotiriou and V. Faraoni, Rev. Mod. Phys. 82, 451 (2010); F. S. N. Lobo, arXiv:0807.1640; S. Capozziello and V. Faraoni, Beyond Einstein Gravity, Fundamental Theories of Physics Vol. 170 (Springer Ed., Dordrecht, 2011).

[2] C. Lanczos, Z. Phys. 73, 147 (1932); Ann. Math. 39, 842 (1938); D. Lovelock, J. Math. Phys. (N.Y.) 12, 498 (1971).

[3] G. Cognola, E. Elizade, S. Nojiri, S. D. Odintsov, and S. Zerbini, Phys. Rev. D 73, 084007 (2006); S. Nojiri and S. D. Odintsov, Phys. Lett. B 631, 1 (2005); Phys. Rev. D 68, 123512 (2003); E. Elizalde, R. Myrzakulov, V. V. Obukhov, and D. Sáez-Gómez, Classical Quantum Gravity 27, 095007 (2010); R. Myrzakulov, D. Sáez-Gómez, and A. Tureanu, Gen. Relativ. Gravit. 43, 1671 (2011); A. de la CruzDombriz and D. Sáez-Gómez, Classical Quantum Gravity 29, 245014 (2012).
[4] J. Alcaraz, J. A. R. Cembranos, A. Dobado, and A. L. Maroto, Phys. Rev. D 67, 075010 (2003); P. Achard et al., Phys. Lett. B 597, 145 (2004); J. A. R. Cembranos, A. Dobado, and A. L. Maroto, Phys. Rev. Lett. 90, 241301 (2003); Phys. Rev. D 68, 103505 (2003); AIP Conf. Proc. 670, 235 (2003); Int. J. Mod. Phys. D 13, 2275 (2004); Phys. Rev. D 70, 096001 (2004); 73, 035008 (2006); 73, 057303 (2006); J. Phys. A 40, 6631 (2007); J. A. R. Cembranos, V. Gammaldi, and A. L. Maroto, Phys. Rev. D 86, 103506 (2012); J. Cosmol. Astropart. Phys. 04 (2013) 051; J. High Energy Phys. 09 (2013) 077; Phys. Rev. D 88, 075021 (2013).

[5] D. Z. Freedman, P. van Nieuwenhuizen, and S. Ferrara, Phys. Rev. D 13, 3214 (1976); S. Deser and B. Zumino, Phys. Lett. 62B, 335 (1976); E. Cremmer, B. Julia, and J. Scherk, Phys. Lett. 76B, 409 (1978); L. J. Hall, J. D. Lykken, and S. Weinberg, Phys. Rev. D 27, 2359 (1983); N. Ohta, Prog. Theor. Phys. 70, 542 (1983); L. AlvarezGaume, J. Polchinski, and M. B. Wise, Nucl. Phys. B221, 495 (1983); H. P. Nilles, Phys. Rep. 110, 1 (1984); 
J. A. R. Cembranos, J. L. Feng, A. Rajaraman, and F. Takayama, Phys. Rev. Lett. 95, 181301 (2005); AIP Conf. Proc. 903, 591 (2007); J. A. R. Cembranos, J. L. Feng, and L. E. Strigari, Phys. Rev. Lett. 99, 191301 (2007); Phys. Rev. D 75, 036004 (2007); M. R. Garousi, Phys. Rev. D 87, 025006 (2013).

[6] T. Biswas, T. Koivisto, and A. Mazumdar, J. Cosmol. Astropart. Phys. 11 (2010) 008; T. Biswas, J. A. R. Cembranos, and J. I. Kapusta, Phys. Rev. Lett. 104, 021601 (2010); J. High Energy Phys. 10 (2010) 048; Phys. Rev. D 82, 085028 (2010); T. Biswas, E. Gerwick, T. Koivisto, and A. Mazumdar, Phys. Rev. Lett. 108, 031101 (2012); T. Biswas, A. S. Koshelev, A. Mazumdar, and S. Y. Vernov, J. Cosmol. Astropart. Phys. 08 (2012) 024.

[7] V. A. Kostelecky and S. Samuel, Phys. Rev. D 39, 683 (1989); D. Colladay and V. A. Kostelecky, Phys. Rev. D 55, 6760 (1997); J. R. Ellis, N.E. Mavromatos, and D. V. Nanopoulos, Phys. Rev. D 61, 027503 (1999); J. Alfaro, H. A. Morales-Tecotl, and L. F. Urrutia, Phys. Rev. Lett. 84, 2318 (2000); G. Amelino-Camelia, Nature (London) 418, 34 (2002); J. Magueijo and L. Smolin, Phys. Rev. Lett. 88, 190403 (2002); J. A. R. Cembranos, A. Rajaraman, and F. Takayama, arXiv:hep-ph/0512020; Europhys. Lett. 82, 21001 (2008); S. Ghosh and P. Pal, Phys. Rev. D 75, 105021 (2007).

[8] L. H. Ford, Phys. Rev. D 40, 967 (1989); J. Beltrán Jiménez and A. L. Maroto, Phys. Rev. D 78, 063005 (2008); J. Cosmol. Astropart. Phys. 03 (2009) 016; Phys. Rev. D 80, 063512 (2009); T. Koivisto and D. F. Mota, J. Cosmol. Astropart. Phys. 08 (2008) 021; J. A. R. Cembranos, C. Hallabrin, A. L. Maroto, and S. J. Núñez Jareño, Phys. Rev. D 86, 021301 (2012); Phys. Rev. D 87, 043523 (2013); J. Cosmol. Astropart. Phys. 03 (2014) 042.

[9] G. W. Horndeski, Int. J. Theor. Phys. 10, 363 (1974); J. D. Bekenstein, Phys. Rev. D 48, 3641 (1993); J. A. R. Cembranos, A. Dobado, and A. L. Maroto, Phys. Rev. D 65, 026005 (2001); J. Cosmol. Astropart. Phys. 10 (2008) 039; Phys. Rev. D 83, 083507 (2011); 84, 083522 (2011); 85, 043505 (2012); J. A. R. Cembranos and L. E. Strigari, Phys. Rev. D 77, 123519 (2008); M. Zumalacarregui, T. S. Koivisto, D. F. Mota, and P. Ruiz-Lapuente, J. Cosmol. Astropart. Phys. 05 (2010) 038; T. S. Koivisto, D. F. Mota, and M. Zumalacarregui, Phys. Rev. Lett. 109, 241102 (2012); Phys. Rev. D 87, 104030 (2013).

[10] C. Brans and R. H. Dicke, Phys. Rev. 124, 925 (1961); C. H. Brans, Phys. Rev. 125, 2194 (1962); J. García-Bellido, A. Linde, and D. Linde, Phys. Rev. D 50, 730 (1994); J. A. R. Cembranos, A. de la Cruz Dombriz, and L. O. Garcia, Phys. Rev. D 88, 123507 (2013).

[11] J. A. R. Cembranos, K. A. Olive, M. Peloso, and J.-P. Uzan, J. Cosmol. Astropart. Phys. 07 (2009) 025.

[12] G. Aad et al. (ATLAS Collaboration), Phys. Lett. B 716, 1 (2012).

[13] S. Chatrchyan et al. (CMS Collaboration), Phys. Lett. B 716, 30 (2012).

[14] P. Jordan, Nature (London) 164, 637 (1949); M. Fierz, Helv. Phys. Acta 29, 128 (1956); C. Brans and R. Dicke, Phys. Rev. 124, 925 (1961); P. G. Bergmann, Int. J. Theor. Phys. 1, 25 (1968); K. Nordtvedt, Astrophys. J. 161, 1059 (1970); R. Wagoner, Phys. Rev. D 1, 3209 (1970).
[15] J. Polchinsky, String Theory (Cambridge University Press, Cambridge, England, 1998).

[16] K. S. Stelle, Gen. Relativ. Gravit. 9, 353 (1978); A. A. Starobinsky, Phys. Lett. 91B, 99 (1980); M. B. Mijíc, M. S. Morris, and W. M. Suen, Phys. Rev. D 34, 2934 (1986); S. M. Carroll, V. Duvvuri, M. Trodden, and M. S. Turner, Phys. Rev. D 70, 043528 (2004); N. Goheer, J. Larena, and P. K. S Dunsby, Phys. Rev. D 80, 061301 (2009); S. Carloni, P. K. S. Dunsby, and A. Troisi, Phys. Rev. D 77, 024024 (2008); K. N. Ananda, S. Carloni, and P. K. S. Dunsby, Phys. Rev. D 77, 024033 (2008); Classical Quantum Gravity 26, 235018 (2009); B. M. Nunez, J. A. R. Cembranos, and A. de la Cruz-Dombriz, AIP Conf. Proc. 1458, 491 (2011); Int. J. Geom. Methods Mod. Phys. 11, 1450001 (2014); A. Abebe, M. Abdelwahab, A. de la CruzDombriz, and P. K. S. Dunsby, Classical Quantum Gravity 29, 135011 (2012); A. Abebe, A. de la Cruz-Dombriz, and P. K. S. Dunsby, Phys. Rev. D 88, 044050 (2013); A. de la Cruz-Dombriz, P. K. S. Dunsby, V.C. Busti, and S. Kandhai, Phys. Rev. D 89, 064029 (2014).

[17] J. A. R. Cembranos, Phys. Rev. D 73, 064029 (2006).

[18] J. A. R. Cembranos, Phys. Rev. Lett. 102, 141301 (2009).

[19] M. Atkins and X. Calmet, Phys. Lett. B 697, 37 (2011); G. Chakravarty, S. Mohanty, and N. K. Singh, Int. J. Mod. Phys. D 23, 1450029 (2014); I. Oda, Phys. Rev. D 87, 065025 (2013); Phys. Lett. B 724, 160 (2013); Adv. Stud. Theor. Phys. 8, 215 (2014); R. Kallosh and A. Linde, J. Cosmol. Astropart. Phys. 06 (2013) 027; 06 (2013) 028; 07 (2013) 002; I. Bars, P. Steinhardt, and N. Turok, Phys. Lett. B 726, 50 (2013); Phys. Rev. D 89, 043515 (2014); M. P. Hertzberg, Phys. Lett. B 745, 118 (2015); R. Costa and H. Nastase, J. High Energy Phys. 06 (2014) 145; J. Ren, Z. Z. Xianyu, and H. J. He, J. Cosmol. Astropart. Phys. 06 (2014) 032; G. K. Chakravarty and S. Mohanty, Phys. Lett. B 746, 242 (2015); I. Oda and T. Tomoyose, J. High Energy Phys. 09 (2014) 165.

[20] J. D. Barrow, Mon. Not. R. Astron. Soc. 184, 677 (1978); J. Yang, D. N. Schramm, G. Steigman, and R. T. Rood, Astrophys. J. 227, 697 (1979); F. S. Accetta, L. M. Krauss, and P. Romanelli, Phys. Lett. B 248, 146 (1990).

[21] J. D. Barrow, Mon. Not. R. Astron. Soc. 184, 677 (1978); K. Arai, M. Hashimoto, and T. Fukui, Astron. Asrophys. 179, 17 (1987); F. S. Accetta, L. M. Krauss, and P. Romanelli, Phys. Lett. B 248, 146 (1990); T. Damour and C. Gundlach, Phys. Rev. D 43, 3873 (1991); J. A. Casas, J. GarciaBellido, and M. Quiros, Mod. Phys. Lett. A 07, 447 (1992); Phys. Lett. B 278, 94 (1992); T. Clifton, J. D. Barrow, and R. J. Scherrer, Phys. Rev. D 71, 123526 (2005).

[22] A. Serna and J. M. Alimi, Phys. Rev. D 53, 3074 (1996); 53, 3087 (1996).

[23] D. I. Santiago, D. Kalligas, and R. V. WagonerPhys. Rev. D, 56, 7627 (1997).

[24] T. Damour and B. Pichon, Phys. Rev. D 59, 123502 (1999).

[25] A. Coc, K. A. Olive, J. P. Uzan, and E. Vangioni, Phys. Rev. D 73, 083525 (2006); 79, 103512 (2009).

[26] A. Riazuelo and J.-P. Uzan, Phys. Rev. D 66, 023525 (2002); 62, 083506 (2000).

[27] C. Schimd, J.-P. Uzan, and A. Riazuelo, Phys. Rev. D 71, 083512 (2005). 
[28] T. Damour and K. Nordtvedt, Phys. Rev. Lett. 70, 2217 (1993); Phys. Rev. D 48, 3436 (1993).

[29] T. Damour and A. M. Polyakov, Nucl. Phys. B423, 532 (1994).

[30] J. Khoury and A. Weltman, Phys. Rev. Lett. 93, 171104 (2004).

[31] P. Brax, C. van de Bruck, A. C. Davis, J. Khoury, and A. Weltman, Phys. Rev. D 70, 123518 (2004).

[32] K. i. Maeda, Phys. Rev. D 39, 3159 (1989).
[33] F. L. Bezrukov and M. Shaposhnikov, Phys. Lett. B 659, 703 (2008); A. O. Barvinsky, A. Y. Kamenshchik, and A. A. Starobinsky, J. Cosmol. Astropart. Phys. 11 (2008) 021; F. Bezrukov, D. Gorbunov, and M. Shaposhnikov, J. Cosmol. Astropart. Phys. 06 (2009) 029; F. L. Bezrukov, A. Magnin, and M. Shaposhnikov, Phys. Lett. B 675, 88 (2009); F. Bezrukov, Classical Quantum Gravity 30, 214001 (2013). 